\title{
Índices de Seleção para um Rebanho Caracu de Duplo Propósito
}

\section{Sandra Aidar de Queiroz ${ }^{1}$, Luciele Cristina Pelicioni², Breno Felix Silva ${ }^{3}$, Juliana Cristina Sesana ${ }^{4}$, Maria Inez Espagnoli Geraldo Martins ${ }^{5}$, Adhemar Sanches ${ }^{6}$}

RESUMO - Neste trabalho, objetivou-se determinar índices de seleção para um rebanho da raça Caracu de duplo propósito, cujo objetivo de seleção $(\mathrm{H})$ incluiu a venda de bezerros desmamados e a produção de leite. As características que compuseram $\mathrm{H}$ foram: produção total de leite, idade ao primeiro parto, período de serviço, peso à desmama e duração da vida produtiva. Foram propostos dois índices de seleção para H. Os critérios de seleção adotados no índice 1 foram: produção de leite na primeira lactação, primeiro período de serviço, peso à desmama (PD) e perímetro escrotal. No índice 2, foram consideradas as mesmas características, sendo, entretanto, o ganho médio diário do nascimento à desmama empregado como critério de seleção para PD. As análises estatísticas para a obtenção dos componentes de (co)variâncias e das estimativas dos parâmetros genéticos e fenotípicos foram realizadas pelo método da máxima verossimilhança restrita livre de derivada, por um modelo animal (uni e bi-característica). As informações zootécnicas e genealógicas, os preços e as quantidades dos insumos e dos produtos foram obtidos no escritório da empresa estudada. Os custos de produção e as receitas da atividade pecuária foram determinados para o período de 1994 a 2000. A equação de lucro foi tomada em base anual, usando-se valores médios para número de animais por categoria, características biológicas e preços.. ${ }^{1} \mathrm{O}$ valor econômico para cada característica foi obtido pela derivada parcial da função de lucro em relação à característica em questão. Os dois índices econômicos de seleção trariam considerável resposta para o objetivo proposto. Entretanto, o índice 1, que incluiu $\mathrm{PD}$, seria um pouco mais eficiente em termos de resposta à seleção total.

Palavras-chave: índices econômicos de seleção, ganho genético, raça de dupla aptidão, valores econômicos

\section{Selection Indices for a Dual Purpose Breed Caracu}

\begin{abstract}
The aims of this research were to determine economic selection indices in order to reach the target objective for a Caracu cattle nucleus. The breeding objective $(\mathrm{H})$ considered a dual purpose breed producing milk and weaning calves. The biological traits affecting income and expenses considered in $\mathrm{H}$ were: total milk yield, age at first calving, number of days open, weaning weight, and productive lifetime. Two economic selection indices were calculated. The selection criteria included in Index 1 were first lactation milk yield, number of days open from first to second calving, scrotal circumference adjusted to age and weight at 15 months and weaning weight (WW). Index 2 included the same traits but WW was switched by average daily gain from birth to weaning. The statistical analyses to estimate the (co)variances and genetic and phenotypic parameters were performed by derivative-free restricted maximum likelihood method, using an animal model (uni and bi-trait). Records on animal performances, pedigrees and prices were provided by the breeder. The production costs and revenues of this herd were calculated from 1994 to 2000 . The profit equation was set to an annual base using annual averages for number of animals per category, biological traits and prices. The economic value of each trait was obtained as the partial derivative of the profit function with respect to that trait (evaluated at the average for all other traits). Both indexes would improve selection response to $\mathrm{H}$, but the economic selection index 1 , including WW, would be a little more efficient for total selection response to $\mathrm{H}$.
\end{abstract}

Key Words: dual purpose breed, economic selection index, economic value, genetic gain.

\section{Introdução}

A raça Caracu é originária do cruzamento nãodirecionado entre os primeiros bovinos trazidos ao Brasil pelos colonizadores. Seus mais prováveis ancestrais são animais das raças da península ibérica
Transtagana, Minhota, Barrosã, Arouquesa, Mirandesa, Brava e Turino (Carvalho Dias, 1948). Os animais denominados Caracu Caldeano apresentam pelagem variando do creme ao vermelho-escuro, bom desenvolvimento corporal, chifres grandes e baixos e boa produção de leite.

\footnotetext{
1 Professor Adjunto - Departamento de Zootecnia - FCAV - UNESP. Bolsista do CNPq. E.mail:saquei@fcav.unesp.br

${ }^{2}$ Doutoranda do Programa de Pós-Graduação em Zootecnia - FCAV - UNESP. Bolsista da FAPESP.

${ }^{3}$ Zootecnista PecMap-Planejamento Agropecuário Ltda.

${ }^{4}$ Zootecnista Agropecuária Jacarezinho- Valparaíso - SP.

5 Professor Assistente Doutor - Departamento de Economia Rural FCAV - UNESP.

6 Professor Adjunto - Departamento de Ciências Exatas - FCAV - UNESP. Via de acesso Prof. Paulo Donato Castellane, Jaboticabal, CEP: 14884-900 - SP.
} 
Sendo uma raça obtida em clima tropical e naturalmente selecionada, o gado Caracu chegou a ser um dos mais criados em todo o país até o início do século $\mathrm{XX}$, perdendo lugar, posteriormente, com a introdução das raças indianas, quando quase chegou à extinção. Por terem sofrido uma rigorosa seleção natural, os reprodutores Caracu adaptaram-se às condições de monta natural a campo no Brasil, de modo que, hoje, é uma das poucas raças com genes de bovinos europeus que suportam servir em monta natural no Brasil Central.

O rebanho Caracu proveniente da Fazenda Chiqueirão, situada no município de Poços de Caldas, MG, foi formado em 1893, com a inserção das primeiras matrizes e dos primeiros touros. A fazenda adquiriu animais até a década de 40, quando, em virtude da diminuição do tamanho da população da raça e da inviabilidade de aquisição de reprodutores externos, o rebanho foi fechado e passou a produzir seus próprios reprodutores, tornando-se o principal reduto de criação e preservação deste recurso genético. Desde a formação deste rebanho, os animais tiveram uma criação mais direcionada à exploração leiteira, uma vez que a raça é considerada de dupla aptidão. Nos últimos anos, a procura por reprodutores para uso em monta natural em sistemas de cruzamento nas condições do Brasil Central tem aumentado consideravelmente, produzindo reflexos no interesse pela seleção também de critérios usados na bovinocultura de corte (Carvalho Dias, 1997).

A seleção simultânea para produção de carne e leite e o desenvolvimento de programas de melhoramento que contemplem ambos os objetivos podem ser importantes para o melhoramento de raças de dupla aptidão (Lôbo et al., 2000a) e parecem ser cruciais para a Caracu, uma vez que a ausência de direção pode levar à deterioração econômica da atividade e à diminuição sensível do tamanho da população. A definição do objetivo de seleção é o primeiro passo para a resolução deste problema e o passo seguinte é a construção de um índice para identificar a lista de características que influenciam o objetivo, determinando a importância relativa de cada característica (Golden et al., 2000).

O objetivo de seleção é, geralmente, representado por uma função contendo receitas e despesas. As equações de lucro podem considerar a empresa como um todo, o animal e sua progênie ou a unidade de produto. Além disso, podem expressar a rentabilidade em base anual ou vitalícia. Entretanto, a descrição das atividades biológicas e econômicas fica defasada, pois despesas e receitas geralmente ocorrem com frequiências e em tempos diferentes durante a vida do animal, para as diferentes características incluídas na função. Por este motivo, alguns autores têm se valido do uso de taxas de juros para remover o efeito do tempo, descontando as despesas e receitas para um ponto fixo da vida do animal, como a data do nascimento ou a data do primeiro parto. Entretanto, segundo Fewson (1993), o aumento da acurácia mediante esta abordagem é limitado quando a equação não inclui rentabilidade vitalícia e, portanto, o desconto pode ser negligenciado, pois não altera significativamente os valores econômicos relativos das características e, valores relativos e não absolutos são necessários na definição do objetivo de melhoramento. Os trabalhos realizados por Ponzoni \& Newman (1989) e Newman et al. (1992) que incluíram fatores de desconto na computação de pesos econômicos comprovaram que estes apresentaram efeitos sobre os valores absolutos, mas apenas efeitos mínimos nos pesos econômicos relativos das características consideradas.

Toda a base genético-econômica para a seleção de várias características simultaneamente foi desenvolvida por Hazel (1943) em seu trabalho clássico de teoria do índice econômico de seleção (TIES). Na TIES, o autor considerou que o valor econômico relativo de cada característica depende da quantidade de aumento no lucro que pode ser esperada para cada unidade de melhoramento naquela característica. Assim, foi introduzido o conceito de genótipo agregado ou objetivo de seleção $(\mathrm{H})$, que foi definido como uma função linear dos valores genéticos ponderados pelos respectivos valores econômicos de cada característica. Dentro desta estrutura, os fatores de ponderação que representam o desejo econômico das mudanças nos critérios de seleção do rebanho (valores econômicos) são combinados com os fatores de ponderação genéticos, representando o quanto as práticas de seleção podem causar melhorias em cada característica (Amer, 1994).

De acordo com Bourdon (1997), um índice econômico de seleção pode ser entendido como a combinação de fatores de ponderação e informações genéticas, no caso clássico, de valores fenotípicos, de mais de uma característica. Os índices são usados na seleção de características múltiplas para a predição do valor genético agregado. As características incluídas no índice, denominadas critérios de seleção ou características indicadoras, devem ser aquelas que 
podem ser mensuradas com facilidade, baixo custo e mais cedo na vida dos animais, além de apresentarem estimativas de herdabilidade de mediana a alta, contribuírem e estarem relacionadas ao objetivo de seleção.

Bourdon \& Golden (2000) argumentam que as características que devem constar do objetivo de seleção podem ser relacionadas nas seguintes categorias: 1) sobrevivência; 2) fertilidade/longevidade; 3) consumo de alimentos; 4) produtos e 5) custos não relacionados com alimentação. Segundo estes autores, pode-se adotar como regra que as características que afetam a sobrevivência são as mais economicamente importantes, pois a morte de vacas e de bezerros representa unidades de produção totalmente perdidas. Quanto maior a perda por morte, menor serão as unidades de produto disponíveis para venda. As características ligadas à fertilidade e à longevidade viriam a seguir, porque influenciam a composição do produto, isto é, a proporção de unidades nas diferentes categorias de animais. $\mathrm{O}$ aumento da fertilidade, por exemplo, resultaria em aumento de vendas de bezerros, enquanto o da longevidade, na diminuição da taxa de descarte de vacas. As próximas, que teriam mesma importância relativa, seriam aquelas relacionadas ao produto e ao consumo de alimentos, uma vez que estas duas categorias possuem conjunto de características similares. Por último, viriam as características não relacionadas aos custos de alimentação, como dificuldade de parto, integridade, temperamento, duração da ordenha, tipo, que, apesar de não apresentarem grande importância econômica, podem acarretar diferenças na satisfação que o produtor tem em seu trabalho.

Segundo Weller (1994), deve-se seguir três princípios básicos para a escolha dos critérios de seleção: 1) todas as características mensuradas que tenham correlações genéticas com as características incluídas no objetivo de seleção devem ser usadas como critérios; 2) geralmente, é mais vantajoso computar as avaliações genéticas das características separadamente; e 3) não devem ser utilizadas, como critérios de seleção, características que sejam razão entre outras medidas.

Desta forma, no objetivo de seleção $(\mathrm{H})$, devem aparecer as características que são economicamente importantes, isto é, relacionadas às receitas e às despesas da empresa, enquanto, no índice econômico de seleção (I), são incluídas aquelas que podem ser medidas de forma fácil e barata e que contribuam ou estejam correlacionadas com H. Características econômicas importantes, mas de avaliação difícil, cara ou problemática podem ser substituídas no I por características indicadoras (Bourdon, 1997). Procura-se construir um índice cujos valores $\mathrm{b}_{i}$ possam maximizar a resposta no objetivo de seleção (genótipo agregado, $\mathrm{H})$, o que equivale dizer que se buscam valores de $b_{i}$ que maximizem a correlação entre $\mathrm{I}$ e $\mathrm{H}$ ou, que minimizem a variância dos erros preditos (Var (H-I).

O objetivo, neste trabalho, foi determinar índices de seleção para o objetivo econômico de produção de leite e venda de bezerros desmamados em bovinos Caracu.

\section{Material e Métodos}

Foram utilizados registros contábeis e de desempenho de um rebanho de bovinos Caracu. Neste estudo, a ordenha foi feita manualmente, duas vezes ao dia, com o bezerro ao pé da vaca deixando-se o leite de uma teta disponível para os bezerros. Os retiros acomodaram de 36 a 75 vacas e o período de lactação foi de, aproximadamente, 300 dias, ocasião em que realizou-se a desmama.

Os animais foram mantidos em pastagens nos meses mais quentes do ano (outubro a maio) e, nos meses mais frios, quando as geadas eram frequientes e a maioria das pastagens piorava em qualidade, foi fornecida silagem de milho (10 kg de matéria verde/ animal/dia) para todos os animais. Todas as vacas em lactação receberam $2 \mathrm{~kg}$ de ração no cocho após a primeira ordenha, independentemente do nível de produção de leite. Os bezerros em amamentação receberam ração no cocho cerca de sessenta dias após o nascimento, fornecendo-se $1,0 \mathrm{~kg}$ de ração por bezerro/dia, composta por farelo de trigo, milho desintegrado com palha e sabugo e torta de algodão.

O manejo reprodutivo consistiu-se de monta natural, com um touro para cada retiro, com cerca de 50 vacas, e cada lote de, aproximadamente, 30 novilhas. A estação de monta foi longa (abril a fevereiro do ano seguinte), objetivando-se manter a produção de leite constante. As novilhas, até 1995, foram colocadas em reprodução com aproximadamente 24 meses de idade. Após 1995, passaram a entrar em reprodução aos 17 meses e cerca de $350 \mathrm{~kg}$. Os touros iniciaram monta com, aproximadamente, 20 meses de idade. Por ser um rebanho fechado desde 1943, foram evitados acasalamentos estreitos, utilizando-se touros de até cinco anos de idade, que permaneciam no 
rebanho até que se obtivessem dados de filhas que confirmassem seu potencial genético. Eventualmente, estes touros serviam em outros plantéis enquanto aguardavam suas avaliações genéticas. No caso das novilhas, o acasalamento foi realizado com touros com o menor grau de parentesco possível.

A seleção dos animais, até 1996, foi realizada com base na produção da mãe e no desenvolvimento do animal, aos 10 meses e aos dois anos de idade, para os machos, e aos dois anos, para as fêmeas. Atualmente, procura-se aproveitar melhor o potencial das novilhas e descartar maior número de vacas após o primeiro parto, tendo como causa a baixa produção de leite. $\mathrm{O}$ descarte de animais adultos baseia-se na produção leiteira, conformação, sanidade e idade.

As características consideradas em $\mathrm{H}$ visaram contemplar as várias atividades biológicas envolvidas na produção de bovinos de dupla aptidão, sejam elas características ligadas à produção de carne e de leite, à reprodução ou à longevidade produtiva. Em $\mathrm{H}$, foram incluídos: a produção total de leite (PL), o período de serviço (PS), a idade ao primeiro parto (IPP), o peso à desmama (PD) e a duração da vida produtiva (VU). As fontes de receita foram a venda dos seguintes itens: bezerros desmamados, novilhas, leite, vacas de descarte e touros de descarte. Os custos com alimentação, sanidade e reprodução foram as principais fontes de despesa. Desenvolveu-se uma equação de lucro anual, sendo o lucro definido como a diferença entre receitas e despesas. A equação de lucro foi feita em base anual, usando-se as médias anuais no período de 1994 a 2000 para o número de animais por categoria, características biológicas e preços. Os valores econômicos (VE) estimados para as características do objetivo de seleção foram: $\mathrm{R} \$ 136,18 ; \mathrm{R} \$ 368,83 ; \mathrm{R} \$-59,46 ; \mathrm{R} \$-51,55$ e $\mathrm{R} \$ 10.016,67$, respectivamente para PL em $\mathrm{kg}$, PD em $\mathrm{kg}$, IPP em dias, PS em dias e VU em anos. O objetivo de seleção, os valores de despesas e receitas, os valores médios das características e do número de animais por categoria foram descritos por Queiroz et al. (2002).

As características biológicas estudadas foram separadas em dois grupos: as componentes do objetivo de seleção, para as quais foram determinados os valores econômicos e os critérios de seleção, para os quais foram estimados fatores de ponderação para que compusessem índices econômicos de seleção (I). Foram analisados dois índices econômicos de seleção para H. As características comuns usadas nos índices foram: produção total de leite na primeira lactação (PL1), como critério para PL e VU, primeiro período de serviço (PS1), como critério para PS e VU e perímetro escrotal aos 15 meses (PE) como critério para IPP. No índice 1 (I1), foi utilizado PD e no índice 2 (I2), o ganho médio diário pré-desmama (GMD) como critério de seleção para PD.

$\mathrm{O}$ arquivo geral de dados após consistência e eliminações, passou a contar com 13.712 registros referentes a lactações de 1973 a 1997 e cerca de 5000 animais no arquivo de genealogia. A formação e editoração dos arquivos e as análises estatísticas preliminares foram feitas no programa computacional SAS (1996). As análises estatísticas para a obtenção dos componentes de (co)variâncias e parâmetros genéticos e fenotípicos foram realizadas pelo método da máxima verossimilhança restrita livre de derivada, por modelos animais uni-característica para estimar as herdabilidades e bi-características para a obtenção das correlações genéticas e fenotípicas, por intermédio do programa MTDFREML (Boldman al., 1993). Os valores econômicos para cada característica foram obtidos pela derivada parcial da equação de lucro com respeito a cada característica do objetivo de seleção (Moav \& Hill, 1966; Harris, 1970).

O sistema de produção, o custo de produção, a obtenção das estimativas dos parâmetros genéticos e fenotípicos das características componentes de $\mathrm{H}$ e dos índices, a equação de lucro utilizada e a derivação dos valores econômicos foram descritos por Queiroz (2002) e Queiroz et al. (2002).

A partir do conhecimento dos valores econômicos das características incluídas em $\mathrm{H}$, pode-se determinar índices econômicos de seleção (I) utilizando-se outras características que não necessariamente aquelas constantes do objetivo. Os I foram determinados de acordo com os preceitos da TIES de Hazel (1943). As etapas necessárias para a obtenção dos coeficientes de regressão de I foram detalhadas por Dekkers (2001) e são apresentadas a seguir.

O objetivo de seleção ou genótipo agregado, representado por $\mathrm{H}$, e definido como uma função dos valores genéticos aditivos dos indivíduos e dos valores econômicos das características de interesse, é dado por:

$$
H=v_{1} g_{1}+v_{2} g_{2} \ldots v_{n} g_{n}=\mathbf{v}^{\prime} \mathbf{g}
$$


em que, $g_{i}$ é o valor genético aditivo da $i^{\text {ésima }}$ característica e $v_{i}$ é o valor econômico desta mesma característica. Em notação matricial, tem-se: $\mathbf{v}^{\prime}=\left[v_{1}, v_{2}, \ldots, v_{n}\right]$ e $\mathbf{g}^{\prime}=\left[g_{1}, g_{2}, \ldots, g_{n}\right]$.

Na prática, os valores genéticos das várias características não são conhecidos. Entretanto, os desempenhos dos animais podem ser medidos ou avaliados e as observações destas características podem ser combinadas em um índice de seleção, I, de forma que:

$$
I=b_{1} x_{1}+b_{2} x_{2} \ldots b_{m} x_{m}=\mathbf{b}^{\prime} \mathbf{x}
$$

em que, $x_{i}$ é uma observação da $i^{\text {ésima }}$ característica e $b_{i}$ é o coeficiente do índice de seleção ou o ponderador para esta mesma característica. Em notação matricial, tem-se que: $\mathbf{b}^{\prime}=\left[b_{1}, b_{2}, \ldots, b_{m}\right]$ e $\mathbf{x}$ ' $=\left[x_{1}, x_{2}, \ldots, x_{m}\right]$.

A meta é estimar os coeficientes do índice de seleção, $b_{i}$, de modo que a seleção de indivíduos baseada nos valores de seus índices de seleção, I, maximize a resposta no genótipo agregado, $\mathrm{H}$ :

$$
\mathbf{b}=\mathbf{P}^{-\mathbf{1}} \mathbf{G v}
$$

em que, Pé a matriz $m x m$ de (co)variâncias fenotípicas das características de I e G é a matriz $m x n$ de (co)variâncias genéticas aditivas entre as características incluídas em $\mathrm{H}$ e em I. Caso as mesmas características constem em $\mathrm{H}$ e em I, a matriz $\mathrm{G}$ terá ordem $m \times m$ e será composta pelas (co)variâncias genéticas aditivas entre as características de $\mathrm{H}$.

A predição da variância do índice $\left(\sigma_{I}^{2}\right)$ permite a comparação entre diferentes possibilidades de índices de seleção e é dada por:

$$
\sigma_{I}^{2}=\mathbf{b}^{\prime} \mathbf{P b}
$$

Esta equação prediz a variância para qualquer vetor arbitrário $b$ usado e não somente para o vetor contendo valores ótimos de $\mathrm{b}$, derivado usando-se a TIES. Porém, se $b$ for o conjunto ótimo de coeficientes de I, então, $\mathbf{b}=\mathbf{P}^{-1} \mathbf{G v}$, que substituído em (4) resultará:

$$
\sigma_{I}^{2}=\mathbf{b}^{\prime} \mathbf{P} \mathbf{P}^{-1} \mathbf{G v}=\mathbf{b}^{\prime} \mathbf{G v}
$$

Esta segunda maneira é a mais conhecida para expressar a variância do índice de seleção, entretanto, deve-se salientar que ela só será verdadeira para um índice de seleção ótimo.
Aplicando-se a equação geral de resposta à seleção $\left(S_{H}\right)$, sendo a seleção baseada em I para melhorar $\mathrm{H}$, pode-se obter a predição da superioridade genética para o genótipo agregado, $\mathrm{H}$, à seleção praticada sobre o índice, I, por:

$$
S_{H}=i r_{H I} \sigma_{H}
$$

em que $i$ é a intensidade de seleção praticada, $r_{H I}$ é a acurácia da seleção baseada em I e $\sigma_{H}$ é o desviopadrão de H. Os valores da equação (6) são dados por:

$$
\sigma=\mathbf{v}^{\prime} \mathbf{C v}
$$

em que $\mathrm{C}$ é a matriz $n \times \mathrm{x} n$ das (co)variâncias genéticas entre as caraterísticas do genótipo agregado. De maneira similar, pode-se calcular a covariância entre $\mathrm{H}$ e I, por:

$$
\sigma_{H I}=\mathbf{b} \mathbf{G} \mathbf{v}
$$

e a correlação entre H e I, por:

$$
\begin{aligned}
r_{H I}=\frac{\sigma_{H I}}{\sigma_{I} \sigma_{H}} & =\frac{\mathbf{b}^{\prime} \mathbf{G v}}{\sqrt{\mathbf{b}^{\prime} \mathbf{P} \mathbf{b} \mathbf{v}^{\prime} \mathbf{C v}}} \\
\mathrm{e} \quad S_{H} & =i \frac{\mathbf{b}^{\prime} \mathbf{G v}}{\sqrt{\mathbf{b}^{\prime} \mathbf{P b}}}
\end{aligned}
$$

As derivações apresentadas em (6) a (10) são válidas para qualquer I e não só para o índice ótimo. Porém, se $\mathrm{b}$ for o conjunto ótimo de coeficientes do índice, em que, $\mathbf{b}=\mathbf{P}^{-1} \mathbf{G v}$, então, como apresentado nas equações (4) e (5), b'Pb = b'Gv e, substituindose na equação (10), tem-se:

$$
S_{H}=i \sqrt{\mathbf{b}^{\prime} \mathbf{G v}}
$$

A resposta pode, ainda, ser obtida diretamente por:

$$
S_{H}=i b_{H I} \sigma_{I}
$$

Para o índice de seleção ótimo, pode-se assumir que $b_{H I}=1$, então:

$$
S_{H}=i \sigma_{I}
$$

Substituindo-se o valor da variância do índice ótimo, definido em (5), tem-se que: 


$$
S_{H}=i \sqrt{\mathbf{b}^{\prime} \mathbf{G v}}
$$

A acurácia do índice $\left(r_{H I}\right)$, definida como a correlação existente entre o índice e o genótipo agregado, é obtida por:

$$
r_{H I}=\sqrt{\frac{\mathbf{b}^{\prime} \mathbf{G v}}{\mathbf{v}^{\prime} \mathbf{C v}}}
$$

A mudança esperada da $i^{\text {ésima }}$ característica $\left(S_{g}\right)$ do genótipo agregado resultante da seleção praticada em I, pode ser calculada por:

$$
S_{g i}=i b_{g i I} s_{I}=i \frac{\sigma_{g_{i} I}}{\sigma_{I}}
$$

em que:

$$
\sigma_{g_{i} I}=\operatorname{cov}\left(g_{i}, \mathbf{b}^{\prime} \mathbf{x}\right)=\mathbf{b}^{\prime} \operatorname{cov}\left(g_{i}, \mathbf{x}\right)=\mathbf{b}^{\prime} \mathbf{G}_{i}
$$

sendo que, $\mathbf{G}_{\boldsymbol{i}}$ representa a $i$ ésima coluna da matriz $\mathbf{G}$, sendo $\mathbf{G}=\left[\mathbf{G}_{1}, \mathbf{G}_{2}, \ldots, \mathbf{G}_{\mathrm{i}}, \ldots, \mathbf{G}_{\mathrm{n}}\right]$

Sendo:

$$
S_{g i}=i \frac{\mathbf{b}^{\prime} \mathbf{G}_{i}}{\sqrt{\mathbf{b}^{\prime} \mathbf{P b}}}
$$

a solução para todos os índices. Para o índice ótimo, a solução também pode ser:

$$
S_{g i}=i \frac{\mathbf{b}^{\prime} \mathbf{G}_{i}}{\sqrt{\mathbf{b}^{\prime} \mathbf{G v}}}
$$

O vetor de resposta à seleção para cada característica de $\mathrm{H}$ é obtido, diretamente, a partir da equação (17), por:

$$
\boldsymbol{S}_{g}=\left[S_{g_{1}}, \ldots, S_{g_{i}}, \ldots, S_{g_{n}}\right]=i \frac{\mathbf{b}^{\prime} \mathbf{G}}{\sqrt{\mathbf{b}^{\prime} \mathbf{P b}}}
$$

Para a solução dos sistemas e a obtenção dos coeficientes de regressão dos índices e dos ganhos genéticos esperados, utilizou-se o procedimento IML do pacote estatístico SAS (1996).

\section{Resultados e Discussão}

Nas Tabelas 1 e 2, constam os valores estimados para os coeficientes de regressão dos índices econômicos de seleção 1 (I1) e 2 (I2), o ganho genético total esperado $\left(\mathrm{R}_{\mathrm{T}}\right)$ e o ganho genético por característica $\left(R_{g}\right)$, após uma geração de seleção, para o objetivo de seleção proposto, isto é, produção de leite e venda de bezerros desmamados aos 12 meses $(\mathrm{H})$. As respostas individuais para cada característica de $\mathrm{H}\left(\mathrm{R}_{\mathrm{g}}\right.$, para $i$ $=1,00)$ podem ser entendidas, também, como os valores genéticos médios estimados (VG) dos reprodutores produzidos após a aplicação da seleção, pois para a seleção fenotípica baseada no desempenho dos indivíduos, o ganho genético é igual ao diferencial de seleção multiplicado pela herdabilidade da característica e pela intensidade de seleção $i$. Se $i=1,00$, o ganho genético torna-se igual ao valor genético, expresso como desvio da média da característica.

A escolha dos critérios de seleção incluídos nos índices foi baseada nas estimativas dos parâmetros genéticos das covariâncias existentes e na possibilidade de as características serem mensuradas mais cedo na vida do animal.

Em I1, foram usados como critérios de seleção as características PL1, PD, PE e PS1. A correlação estimada entre o objetivo e o índice de seleção foi igual a 0,7603, o que sugere ser este índice confiável (Tabela 1). Constata-se, ainda, a grande importância das características reprodutivas PE e PS1 para a lucratividade da empresa (5294,70 e -11,21, respectivamente) e, verifica-se também que o valor do coeficiente de regressão do PD foi negativo (-146,05), o que foi ocasionado pelas estimativas negativas das covariâncias genéticas entre PL e PD, e PL1 e PD, sendo que, também, foram desfavoráveis as correlações genéticas estimadas entre PS1 e PD e PS e PD. Em H, o valor econômico de PL foi expressivo e, portanto, o índice aplicou ponderação negativa para PD. A lucratividade média esperada, supondo-se intensidade de seleção praticada igual a 1, foi de $\mathrm{R} \$ 27.387,22 /$ geração com a utilização deste índice, que deve ser entendida como acréscimo desta magnitude ao lucro médio anual da empresa. A resposta à seleção seria ainda maior $(\mathrm{R} \$ 36.151,13)$ se fosse utilizada intensidade de seleção igual a 1,32, que é a praticada para os machos deste rebanho. Os ganhos genéticos esperados em cada característica componente do objetivo de seleção após uma geração de seleção, considerando-se $i=1,00$ seriam iguais a $+167,7232 \mathrm{~kg}$ de leite; $-0,9736 \mathrm{~kg}$ de PD; 2,4313 dias de IPP; +4,2688 dias de PS e +0, 4972 anos de vida produtiva, expressos como desvios da média do rebanho. Estes resultados indicam que a seleção baseada em I1, aplicando-se um diferencial de seleção padronizado, resultaria na produção de reprodutores com estes valores genéticos médios estimados (VG). Os VG esperados destes reprodutores para o mérito genético total seriam iguais a $\mathrm{R} \$ 27.387,22$; se $i=1,00$ ou $\mathrm{R} \$ 36.151,13$; se 
Tabela 1 - Critérios de seleção, coeficientes de regressão estimados para o índice de seleção 1 (I1), resposta total esperada à seleção $\left(R_{T}\right)$ e resposta à seleção esperada por característica $\left(R_{g}\right)$ do objetivo de seleção de produção de leite e venda de bezerros aos 12 meses $(\mathrm{H})$, para um rebanho Caracu

Table 1 - Selection criteria, regression coefficients to selection index 1 (I1), total predicted response to selection $\left(R_{T}\right)$ and predicted response to selection to each trait $\left(R_{G}\right)$ of the selection objective of selling milk and weaned calves $(H)$, for a Caracu Herd

Coeficientes de regressão (b)

Regression coefficients $(b)$

\begin{tabular}{|c|c|c|c|c|c|}
\hline \multirow{2}{*}{$\begin{array}{c}\text { Critérios } \\
\text { Criteria } \\
\end{array}$} & & \multirow[b]{2}{*}{ Acurácia } \\
\hline & PL1 (kg) & PD (kg) & $\mathrm{PE}(\mathrm{cm})$ & PS1 (dias) & \\
\hline $\begin{array}{l}\text { Características } \\
\text { Traits }\end{array}$ & $\mathrm{PL}(\mathrm{kg})$ & $\mathrm{PD}(\mathrm{kg})$ & IPP (dias) & PS (dias) & VU (anos) \\
\hline $\mathrm{R}_{\mathrm{\sigma}} \quad \mathrm{i}=1,00$ & $167,7232 \mathrm{~kg}$ & $-0,9736 \mathrm{~kg}$ & $-2,4313$ dias & 4,2688 dias & 0,4972 anos \\
\hline $\mathrm{R}_{\mathrm{g}}^{\mathrm{g}} \quad \mathrm{i}=1,32$ & $221,2437 \mathrm{~kg}$ & $-1,2843 \mathrm{~kg}$ & $-3,2071$ dias & 5,6310 dias & 0,6559 anos \\
\hline $\mathrm{R}_{\mathrm{T}}^{\mathrm{g}} \mathrm{i}=1,00(\mathrm{R} \$)$ & $\mathrm{R}_{\mathrm{T}} \mathrm{i}=1,32(\mathrm{R} \$)$ & Var. H & d.p. $H(R \$)$ & Var.I1 & d.p. I1 (R\$) \\
\hline $27.382,22$ & $36.151,13$ & 1.297.540.000 & $36.021,38$ & 750.059 .705 & $27.382,22$ \\
\hline
\end{tabular}

$\mathrm{i}=$ intensidade de seleção; $\mathrm{PL}=$ produção total de leite; $\mathrm{PD}=$ peso à desmama; $\mathrm{PP}=$ =idade ao primeiro parto; $\mathrm{PS}=$ período de serviço; $\mathrm{VU}=$ duração da vida produtiva; $\mathrm{PL1}$ =produção total de leite na primeira lactação; PE=perímetro escrotal; PS1=primeiro período de serviço; Var=variância; d.p.=desvio-padrão.

$i=$ selection intensity, $P L=$ total milk yield; $P D=$ weaning weight; $I P P=$ age at first calving; $P S=$ days open; VU = productive lifetime; $P L 1=$ milk yield in first lactation; $P E=$ scrotal circumference; $P S 1=$ first days open; Var = variance; $d . p$. = standard deviation .

$i=1,32$. Pode-se observar, ainda, que a utilização de I1 contemplaria a meta de produzir animais com valores genéticos superiores para PL, IPP e VU, mas levaria a respostas indesejáveis nas características PD e PS (Tabela 1).

A utilização de I2 (Tabela 2), em que o critério de seleção PD foi substituído por GMD, provocaria resultados semelhantes aos apresentados e discutidos para I1, uma vez que as correlações existentes entre GMD e PL e GMD e PL1 apresentaram sentido e magnitude próximas àquelas entre PD e PL e PD e PL1. Porém, ao contrário do ocorrido em I1, as covariâncias genéticas e fenotípicas estimadas entre GMD e PS e GMD e PS1 foram favoráveis. A aplicação deste índice de seleção resultaria em respostas um pouco menores àquelas que seriam obtidas empregando-se I1, para o mérito genético total $\left(\mathrm{R}_{\mathrm{T}}\right)$ e PL e, conseqüentemente, com acurácia também pouco menor $(0,7570)$. Uma possível vantagem do emprego deste índice contendo GMD seria o fato de este critério não levar em conta o peso ao nascer e, por isto, apresentar covariâncias mais baixas com esta característica, prevenindo possíveis problemas de partos distócicos no futuro. Além disso, a utilização de GMD, no índice de seleção, produziria resposta menos desfavorável ao PD (-0,3308 e -0,4363 kg) e ao PS $(+4,0609$ e +5,3567 dias) e mais favorável à IPP $(-2,8092$ e $-3,7056$ dias $)$ e à VU $(+0,5135$ e $+0,6773$ anos), respectivamente para $i=1,00$ e $i=1,32$, que a seleção baseada em I1.

Em trabalhos descritos na literatura, pode-se encontrar indícios de antagonismo genético entre o efeito materno e o direto para características prédesmama (Eler et al., 1994; Eler et al., 1995). Albuquerque et al. (1998) estimaram, em análises uni e bi-características, correlação genética entre produção de leite e ganho médio diário em bovinos da raça Caracu iguais a $-0,22$ e $-0,43$, respectivamente. Robinson (1996), utilizando diferentes modelos para estimar e interpretar os parâmetros genéticos dos efeitos genéticos direto e materno, obteve estimativas de covariância entre efeitos direto e materno negativas para todas as características analisadas. Segundo a autora, os resultados sugeriram que estas estimativas negativas foram mais uma conseqüência da variação adicional entre touros ou touro $\mathrm{x}$ ano, evidência de uma relação genética negativa.

Schaeffer (1996), utilizando um exemplo numérico, demonstrou que a correlação genética negativa entre efeitos direto e materno poderia resultar da estimativa do efeito materno como função linear do efeito direto. Assim, em seu exemplo, o efeito materno foi estimado para touros mesmo que não tivessem qualquer filha desmamando um bezerro. Esses touros, sem filhas, apresentaram correlação entre os efeito direto e materno igual a menos um $(-1,0)$, porque não 
Tabela 2 - Critérios de seleção, coeficientes de regressão estimados para o índice de seleção 2 (I2), resposta total esperada à seleção $\left(R_{T}\right)$ e resposta à seleção esperada por característica $\left(R_{g}\right)$ do objetivo de seleção de produção de leite e venda de bezerros aos 12 meses $(\mathrm{H})$, para um rebanho Caracu

Table 2 - Selection criteria, regression coefficients to selection index 2 (I2), total predicted response to selection $\left(R_{T}\right)$ and predicted response to selection to each trait $\left(R_{G}\right)$ of the selection objective of selling milk and weaned calves $(H)$, for a Caracu Herd

\begin{tabular}{|c|c|c|c|c|c|}
\hline \multirow[b]{2}{*}{$\begin{array}{c}\text { Critérios } \\
\text { Criteria }\end{array}$} & \multicolumn{4}{|c|}{$\begin{array}{l}\text { Coeficientes de regressão (b) } \\
\text { Regression coefficients }(b)\end{array}$} & \multirow[b]{2}{*}{ Acurácia } \\
\hline & $\operatorname{PL1}(\mathrm{kg})$ & GMD (kg/dia) & $\mathrm{PE}(\mathrm{cm})$ & PS1 (dias) & \\
\hline $\begin{array}{l}\text { Índice } 2 \text { (I2) } \\
\text { Index } 2\end{array}$ & 50,3144 & $-8.916,0950$ & $5.353,3350$ & $-33,1467$ & 0,7570 \\
\hline $\begin{array}{l}\text { Características } \\
\text { Traits }\end{array}$ & $\mathrm{PL}(\mathrm{kg})$ & $\mathrm{PD}(\mathrm{kg})$ & IPP (dias) & PS (dias) & $\mathrm{VU}$ (anos) \\
\hline $\begin{array}{l}\mathrm{R}_{\mathrm{g}} \quad \mathrm{i}=1,00 \\
\mathrm{R}_{\mathrm{g}} \quad \mathrm{i}=1,32 \\
\mathrm{R}_{\mathrm{T}} \mathrm{i}=1,00(\mathrm{R} \$) \\
27.268,99\end{array}$ & $\begin{array}{c}163,6740 \mathrm{~kg} \\
215,9024 \mathrm{~kg} \\
\mathrm{R}_{\mathrm{T}} \mathrm{i}=1,32(\mathrm{R} \$) \\
35.970,52\end{array}$ & $\begin{array}{c}-0,3308 \mathrm{~kg} \\
-0,4363 \mathrm{~kg} \\
\text { Var. H } \\
1.297 .540 .000\end{array}$ & $\begin{array}{c}-2,8092 \text { dias } \\
-3,7056 \text { dias } \\
\text { d.p. H (R\$) } \\
36.021,38\end{array}$ & $\begin{array}{c}\text { 4,0609 dias } \\
\text { 5,3567dias } \\
\text { Var.I2 } \\
\text { 743.597.656 }\end{array}$ & $\begin{array}{c}0,5135 \text { anos } \\
0,6773 \text { anos } \\
\text { d.p. I2 (R\$) } \\
27.268,99\end{array}$ \\
\hline
\end{tabular}

$\mathrm{i}$ = intensidade de seleção; $\mathrm{PL}=$ produção total de leite; $\mathrm{PD}=$ peso à desmama; $\mathrm{IPP}=$ idade ao primeiro parto; $\mathrm{PS}=$ período de serviço; $\mathrm{VU}$ = duração da vida produtiva; $\mathrm{PL} 1=$ produção total de leite na primeira lactação; GMD = ganho médio diário do nascimento à desmama; $\mathrm{PE}=$ perímetro escrotal; PS1 = primeiro período de serviço; Var = variância; d.p. = desvio-padrão.

$i=$ selection intensity, $P L=$ total milk yield; $P D=$ weaning weight; $I P P=$ age at first calving; $P S=$ days open; $V U=$ productive lifetime; $P L 1=$ Milk yield in first lactation; $G M D=$ average daily gain form birth to weaning; $P E=$ scrotal circumference; $P S 1=$ first days open; Var = variance; d.p. = standard deviation.

existia nenhuma observação para a estimativa do efeito materno, sendo este função, apenas, do efeito direto. Dentre as muitas considerações acerca das estimativas de efeitos diretos e maternos, Fries \& Albuquerque (1998) concluíram que o melhor seria usar covariância igual a zero para efeitos direto e materno, pelo menos até que se obtenham informações suficientes e que se conheça melhor a relação entre estes efeitos.

Desse modo, os índices 1 (I1*) e 2 (I2*) foram recalculados considerando-se as covariâncias genéticas entre PD e PL, entre PD e PL1 e entre GMD e PL iguais a zero.

Para $\mathrm{I} 1^{*}$, houve redução no valor absoluto do coeficiente de regressão de PD que passou de $-146,0551$ a $-86,5150$ e incremento no de PS1, que variou de $-11,2153$ para $-21,0184$. Os demais valores mantiveram-se próximos aos anteriores, isto é, os coeficientes de regressão estimados para PL1 e PE foram, respectivamente, 51,8764 e 5295,0184. A acurácia manteve-se alta $(0,7594)$ e semelhante à de I1. A seleção baseada nesta variante de I1 produziria resultados práticos muito semelhantes aos do emprego do índice original, isto é, reprodutores com VG médio igual a $\mathrm{R} \$ 27.516,22 /$ geração. Para as características de $\mathrm{H}$, os VG dos animais seriam, também, semelhantes aos do índice 1 original, entretanto, haveria considerável queda na redução do valor genético médio dos animais para PD. Este índice produziria animais com VG iguais a $165,5440 \mathrm{~kg}, \mathbf{- 0 , 0 8 9 5} \mathbf{~ k g}$, - 2, 5525 dias, + 4,1747 dias e 0,5060 anos, respectivamente, para PL, PD, IPP, PS e VU.

Uma variante do índice 2 (I2*), considerando as covariâncias genéticas entre PD e PL e entre GMD e PL iguais a zero, foi elaborada produzindo resultados globais semelhantes aos de I2, isto é, acurácia igual a 0,7581 e resposta à seleção de $\mathrm{R} \$ 27.471,76 /$ geração $(i=1,00)$. Exceto para GMD, os coeficientes de regressão para as características indicadoras foram, do mesmo modo, próximos aos obtidos por I2, isto é, 50,7549 para PL1, 5.312,7811 para PE e -35,7774 para PS1. Porém, diferença considerável na ênfase seletiva ocorreria para o critério de seleção GMD, que passaria a apresentar coeficiente de regressão positivo e, em valor absoluto, muito menor $(844,5916)$, o que resultaria no emprego de reprodutores com VG para PD positivos $(+0,3410 \mathrm{~kg})$. Para as demais características do objetivo de seleção, os VG dos animais seriam próximos aos estimados pelo I2 original, 163,099 kg, -2,8117 dias, +4,0064 dias e 0,5165 anos, para PD, IPP, PS e VU, respectivamente.

Assim como existem questionamentos quanto à natureza da associação entre PD e PL e GMD e PL, o uso do perímetro escrotal como critério de seleção para características reprodutivas é ainda recente, mas seu uso vem sendo recomendado por técnicos e muitos criadores o tem empregado como critério de 
escolha para a aquisição de sêmen e/ou de touros. No presente trabalho, a associação genética entre IPP e PE foi favorável, como relatado na literatura (Toelle \& Robinson, 1985), indicando ser adequado para a predição de precocidade sexual. Entretanto, as correlações genéticas estimadas para esta população, entre PE e PS e entre PE e PS1, foram positivas, embora de magnitude não elevada, isto é, 0,08 e 0,20, respectivamente. As relações existentes entre PE e características que mensuram assiduidade reprodutiva de fêmeas, como intervalo de partos, período de serviço e número de serviços/concepção, foram até o momento pouco estudadas, merecendo o PE atenção e cuidado quando empregado como critério de seleção. Cabe ressaltar, no entanto, que a utilização de IPP como critério de seleção nos índices estudados provavelmente produziria respostas não muito diferentes, uma vez que as covariâncias estimadas entre IPP e PS também foram desfavoráveis, isto é, 55,47691 e $-366,6627$, respectivamente, para as covariâncias genética e ambiental.

Para simplificar a aplicação do índice utilizando os desvios dos valores fenotípicos das características indicadoras, pode-se dividir todos os coeficientes de regressão do índice por um valor comum, por exemplo, o do coeficiente de regressão da PL1. A ênfase seletiva continuará a mesma, mas para a predição da resposta à seleção, o valor obtido deverá ser multiplicado pelo divisor dos coeficientes de regressão do índice.

Os índices econômicos de seleção usando valores fenotípicos não são capazes de levar em conta diferenças genéticas entre rebanhos ou grupos contemporâneos (GC). Portanto, devem ser utilizados somente quando as informações fenotípicas forem provenientes de GC geneticamente similares. Salienta-se que as informações fenotípicas usadas para a construção desses índices devem ser limitadas a um período de tempo relativamente curto, para evitar que a mudança genética não cause diferença acentuada entre os GC. Também deve-se ressaltar que a utilização de índices de seleção para animais de dupla aptidão apresenta a limitação de que os touros candidatos à seleção não expressam seu genótipo para características leiteiras e de corte; que suas progênies expressam os dois grupos de características desigualmente (Lôbo, 1999); e que as características incluídas no índice não acontecem simultaneamente na vida do animal nem com a mesma freqüência, resultando em diferentes contribuições genéticas dos reprodutores à sua descendên- cia. Se esses fatores não forem considerados, podese subestimar a resposta à seleção das características de crescimento e superestimar a das reprodutivas (Newman et al., 1992). Uma maneira de sanar o viés introduzido pelo tempo é levar a expressão de todas as características que compõem o índice para um determinado ponto da vida do animal, como o ano de nascimento, por exemplo, utilizando expressões descontadas (Mcclintock \& Cunningham, 1974). O método do fluxo de genes desenvolvido por Hill (1974) e detalhado por Graser (1993) pode ser utilizado também para computar a contribuição dos reprodutores às suas progênies e os diferentes tempos de permanência das diversas categorias animais no rebanho. Lôbo et al. (2000a e 200b) se valeram desta abordagem para a avaliação de esquemas de seleção de bovinos Guzerá de dupla aptidão.

Outro ponto importante é que os valores econômicos devem ser alterados de tempos em tempos, pois dependem do nível de produção do rebanho, dos preços de insumos e produtos, dos demais fatores de produção e do valor da força de trabalho, levando a alterações nos índices de seleção. A adoção de um horizonte de planejamento de 20 a 25 anos (4 a 5 gerações) para a avaliação do programa de seleção tem sido recomendada (Hill, 1974).

A seleção de múltiplas características adotandose índices econômicos de seleção é, segundo Bourdon (1997), a maneira mais rápida e eficiente de melhorar o valor genético agregado, porque utiliza uma grande quantidade de informação de várias características para produzir um valor único - o valor do índice - que prediz o mérito genético econômico médio de um indivíduo. Ele torna fácil a classificação dos indivíduos, porém não caracteriza o animal em detalhes. Como visto neste trabalho, o índice tenta conciliar a seleção para características que possuem relações genéticas antagônicas, mas o resultado nem sempre favorece o ganho genético para todas as características. O emprego de índices evidencia a direção em que a seleção está sendo processada mas não o alvo a ser atingido. Ocasionalmente, a utilização de índices de seleção pode resultar na seleção de indivíduos extremos, que seriam desaconselháveis para o melhoramento em uma ou mais características (por exemplo, peso ao nascer). Nesta situação, segundo Bourdon (1997), poder-se-ia associar a utilização do índice com outro método de seleção de múltiplas características, como o dos níveis independentes de rejeição, para eliminar estes indivíduos extremos. 
A derivação de valores econômicos e a construção de índices de seleção dependem da coleta e do armazenamento de informações individuais confiáveis e relacionadas às fontes de receitas e de despesas da empresa. Estas informações devem possibilitar a estimação de parâmetros genéticos e fenotípicos acurados para serem usadas na equação de lucro e nos índices de seleção. Características de difícil mensuração, como o consumo de alimento (matéria seca) por animais criados e mantidos em pastagens, são essenciais para o cálculo do valor econômico de características reprodutivas e, por si só, possuem valor econômico de importante mensuração para sistemas de produção menos intensivos. Além disto, o conhecimento da correta associação existente entre as características que serão empregados para compor o objetivo de seleção e o índice é fundamental para que estes possam predizer corretamente a resposta à seleção, especialmente de animais de raças de dupla aptidão, em que é necessário conciliar objetivos, aparentemente, antagônicos. Para elucidar estes pontos, é providencial que as instituições de pesquisa conduzam experimentos que solucionem questões, como: qual é a variabilidade individual existente entre bovinos para consumo de alimento em pastagem; quais as reais associações genéticas entre os efeitos aditivos genéticos direto e materno das características pré-desmama de bovinos e entre as características reprodutivas de fêmeas (IPP, PS, IEP) e de machos (PE), entre outras. Estudos desenvolvidos nesta área serviriam para alicerçar e dar embasamento a novas pesquisas envolvendo trabalhos de avaliação genético-econômica, que, por sua vez, além da aplicação direta dos resultados gerados, podem ser úteis na identificação de áreas em que o conhecimento é deficiente e servirem como base para o planejamento de investigações futuras em bovinocultura.

Os resultados obtidos nesta pesquisa evidenciaram que o melhoramento do desempenho dos animais nas características biológicas estudadas sem incorrer em aumento nos custos ou em mudanças no sistema de produção resultaria em grande impacto no lucro. Neste estudo, as estruturas de covariâncias genéticas e fenotípicas entre as características incluídas nos índices $\mathrm{I} 1$ e $\mathrm{I} 2$ e no objetivo $\mathrm{H}$, aliadas aos valores econômicos estimados para as características, não possibilitaram a obtenção de ganho genético favorável para todas as características. Portanto, seria prudente e conveniente a verificação periódica do objetivo de seleção e a estimação de novos valores econômicos e de novos índices de seleção, caso haja mudanças, destacando-se, que, neste estudo, foram específicos para as situações em que foram obtidos e não devem servir para utilização em outras situações ou em outros rebanhos.

\section{Conclusões}

Os dois índices econômicos de seleção trariam considerável resposta à seleção para o objetivo proposto.

Para o objetivo de seleção considerado, o índice 1, incluindo peso à desmama como critério de seleção, seria um pouco mais eficiente em termos de resposta à seleção total.

A magnitude e o sinal das estimativas de covariâncias genéticas entre os efeitos direto e materno das características de crescimento pré-desmama e produção de leite foram importantes para a predição da resposta à seleção. Portanto, investigações futuras devem ser implementadas para melhorar as estimativas destes efeitos.

\section{Agradecimento}

Ao zootecnista MSc. André Stein Carvalho Dias, pela cessão dos dados utilizados nesta pesquisa.

\section{Literatura Citada}

ALBUQUERQUE, L.G.; QUEIROZ, S.A.; FRIES, L.A. Correlação genética entre produção de leite e crescimento pré desmame em bovinos da raça Caracu. In: REUNIÃO ANUAL DA SOCIEDADE BRASILEIRA DE ZOOTECNIA, 35., 1998, Botucatu. Anais... Botucatu: Sociedade Brasileira de Zootecnia, 1998. p.288-291.

AMER, P.R. Estimation of economic weights in genetics improvement using neoclassical production theory: an alternative to rescaling. Animal Production, v.54, p.341-50, 1994.

BOLDMAN, K.G.; KRIESE, L.A.; Van VLECK, L.D. et al. A manual for use of MTDFREML: a set of programs to obtain estimates of variances and covariance. Lincoln: Department of Agriculture, Agricultural Research Service, 1993.120p.

BOURDON, R.M. Understanding animal breeding. New Jersey: Prentice Hall, 1997. 523p.

BOURDON, R.M.; GOLDEN, B.L. EPDs and economics: determining the relative importance of traits. Colorado, 2000. p. 1-9. Disponível em: 〈http://ansci.colostate.edu/> Acesso em: 18 jan. 2002.

CARVALHO DIAS, E. O gado Caracu sob o sistema de retiros. Revista Ceres, v.7, n.22, p.338-402, 1948.

CARVALHO DIAS, A.S. Estudo de características produtivas e de permanência em um rebanho de bovinos da raça 
Caracu. Jaboticabal: Universidade Estadual Paulista, 1997. 65p. Dissertação (Mestrado em Zootecnia) - Universidade Estadual Paulista, 1997.

DEKKERS, J.C.M. Economic aspects of applied animal breeding programs. Guelph: University of Guelph, 2001. 320p. (Apostila de curso).

ELER, J.P.; FERRAZ, J.B.; LÔBO, R.B. et al. Genetic antagonism between growth and maternal ability in nellore cattle. Revista Brasileira de Genética, v.17, n.1, p.59-64,1994.

ELER, J.P.; Van VLECK, L.D.; FERRAZ, J.B. Estimation of variance due to direct and maternal effects of growth traits of nellore cattle. Journal of Animal Science, v.73, p.32533258, 1995.

FEWSON, D. Definition of the breeding objectives. In: BRAWICK, S.A.; FEWSON, D.; GRASER, H.U. et al. (Eds.) Design of livestock breeding programs. Armidale: University of New England, 1993. p.53-58.

FRIES, L.A.; ALBUQUERQUE, L.G. Pressuposições e restrições dos modelos animais com efeitos maternos em gado de corte. In: PARANHOS DA COSTA, M.J.R.; CROMBERG, V.U. (Eds.) Comportamento materno em mamíferos: bases teóricas e aplicações aos ruminantes domésticos. Jaboticabal: SBEt, 1998. p.179-214.

GOLDEN, B.L.; GARRICK, D.J.; NEWMAN, S.; ENNS, M. Economically relevant traits: a framewok for the next generation of EPDs. Colorado, 2000. p.1-13. Disponível em: <http://ansci.colostate.edu/> Acesso em: 18 jan. 2002.

GRASER, H.U. Gene flow. In: BRAWICK, S.A.; FEWSON, D.; GRASER, H.U. et al. (Eds.) Design of livestock breeding programs. Armidale: University of New England, 1993. p.127-135.

HARRIS, D.L. Breeding for efficiency in livestock production: defining the economic objectives. Journal of Animal Science, v.30, p.860-865, 1970.

HAZEL, L.N. The genetic basis of constructing selection index. Genetics, v.28, p.476-90, 1943.

HILL, W.G. Prediction and evaluation of response to selection with overlapping generations. Animal Production, Amsterdan, v.18, p.117-139, 1974.

LÔBO, R.N.B. Programas de seleção para bovinos zebus de dupla aptidão. Belo Horizonte: Escola de Veterinária. 1999, 76p. Tese (Doutorado) - Universidade Federal de Minas Gerais, 1999.

LÔBO, R.N.B.; MADALENA, F.E.; PENNA, V.M. Avaliação de um esquema de seleção para bovinos Zebus de dupla aptidão. Revista Brasileira de Zootecnia, v.29, n.5, p.1349-1360, 2000a.
LÔBO, R.N.B.; MADALENA, F.E.; PENNA, V.M. Avaliação de esquemas de seleção alternativos para bovinos Zebus de dupla aptidão. Revista Brasileira de Zootecnia, v.29, n.5, p.1361-1370, 2000b.

MCCLINTOCK, A.E.; CUNNINGHAM, E.P. Selection in dual purpose cattle population: definition of breeding objective. Animal Production, v.18, p.237-248, 1974.

MOAV, R.V.; HILL, W.G. Specialized sire and dam lines. IV. Selection within lines. Animal Production, v.8, p.375-90, 1966.

NEWMAN, S.; MORRIS, C.A.; BAKER, R.L. et al. Genetic improvement of beef cattle in New Zealand: breeding objectives. Livestock Production Science, v.32, n. 2, p.111-130, 1992.

PONZONI, R.W.; NEWMAN, S. Developing breeding objectives for australian beef cattle production. Animal Production, v.49, p.35-47, 1989.

QUEIROZ, S.A. Valores econômicos e índices de seleção para características biológicas dos objetivos de seleção de um núcleo de bovinos da raça Caracu. Jaboticabal: Universidade Estadual Paulista, 2002. 194p. Tese (Livre-Docência) Universidade Estadual Paulista. 2002.

QUEIROZ, S.A.; PELICIONI, L.C.; SESANA, J.C. et al. Economic values for biological traits in the breeding objective of Caracu cattle. In: WORLD CONGRESS ON GENETICS APLLIED TO LIVESTOCK PRODUCTION, 7., Montpellier, Abstracts... Montpellier: 2002. p.47 CD-Rom

ROBINSON, D.L. Estimation and interpretation of direct and maternal genetic parameters for weights of Australian Angus cattle. Livestock Production Science, v.45, p.1-11, 1996.

STATISTICAL ANALYSES SYSTEM - SAS. SAS/STAT language guide. version 6. 3.ed. Cary: 1996. 530p.

SCHAEFFER, L.R. Tópicos avançados em melhoramento genético animal. Faculdade de Ciências Agrárias e Veterinárias, 1996. 98 p. (Apostila de curso).

TOELLE, V.D.; ROBISON, O.W. Estimates of genetic correlation between measurements and female reproductive traits in cattle. Journal of Animal Science, v.60, p.89-100, 1985.

WELLER, J.L. Economic aspects of animal breeding. London: Chapman \& Hall, 1994. 244p.

Recebido em: 22/04/03 Aceito em: $28 / 12 / 04$ 